\title{
Macroscopic two-state systems in trapped atomic condensates
}

\author{
Dmitry Solenov* and Dmitry Mozyrsky ${ }^{\dagger}$ \\ Theoretical Division (T-4) and the Center for Nonlinear Studies (CNLS), \\ Los Alamos National Laboratory, Los Alamos, NM 87545, USA
}

(Dated: June 19, 2018)

\begin{abstract}
We consider a macroscopic two-sate system based on persistent current states of a Bose-Einstein condensate (BEC) of interacting neutral atoms confined in a ring with a weak Josephson link. We demonstrate that macroscopic superpositions of different BEC flows are energetically favorable in this system. Moreover, a macroscopic two-state dynamics emerges in the low energy limit. We also investigate fundamental limitations due to the noise inherent to the interacting BEC of Josephsonring geometry. We show that the coherent macroscopic dynamics is readily measurable for an experimentally accessible range of parameters.

PACS numbers: 03.75.Kk, 37.10.Gh, 85.25.Cp
\end{abstract}

Realization of macroscopic quantum two-state systems has been a challenge for cold-atom BEC physics from early stages of its experimental development. The research in this area has been primarily focused on manyparticle dynamics within double-well trapping potential [1, 2]. At the same time, despite an appealing similarity with the microscopic single particle two-state system, this geometry provides no easy way to achieve a superposition of distinct many-body quantum states. Indeed, a collection of non-interacting (or weakly interacting) boson atoms confined in a double-well trap condenses into a BEC with the "product" wave function not suitable to form a macroscopic two-sate configuration. Repulsively interacting particles in such trap favor [3] Fock states - the system enters Mott or "Coulomb blockade"like regime. Hence, attractively interacting particles become the only option in such geometry. In the latter case, atoms correlate [4] forming a "Schrodinger cat" state $\Psi_{\lambda<0}^{D W}=\left[\prod_{i} \psi_{L}\left(\mathbf{r}_{i}\right)+\prod_{i} \psi_{R}\left(\mathbf{r}_{i}\right)\right] / \sqrt{2}$, where $\psi_{L / R}(\mathbf{r})$ denote the single particle states localized the left/right well of the trapping potential. Observation of this macroscopic superposition, however, is extremely challenging. The only manifestation of a coherent superposition is the presence of the off-diagonal matrix elements in the macroscopic two-state basis. In the case of the doublewell trapping potential these matrix elements are proportional to the probability of all $N$ particles to tunnel through the barrier, which is extremely small.

In this paper we study a cold atom based macroscopic two-state system (a qubit) based on a persistent current BEC-Josephson system [5]. The two macroscopic states are metastable current-carrying states of a BEC confined in a Josephson ring trap [6, 7]. Such systems have become experimentally available due to recent successes in dynamical BEC trapping [8]. We start by deriving an effective Schrodinger equation describing the low-lying

\footnotetext{
*E-mail: solenov@lanl.gov

${ }^{\dagger}$ E-mail: mozyrsky@lanl.gov
}

energy states of the system in terms of the phase difference across the Josephson junction. For that we introduce a simple anzats that parameterizes the low-lying states as a superposition of the condensate states with different phases. Then we consider a better ground state anzats that accounts for the fluctuations in the systems arising from the interparticle interactions. We briefly discuss their influence on system's dynamics and then analyze their effect on the detection of the systems's state. Particularly, we show that the admixture of "noncondensate" particles acts as an effective noise in the time-of-flight (TOF) images of the BEC and therefore imposes limitations on the resolution of such measurements. We briefly discuss these fundamental limitations and argue that weakly coupled Bose systems provide a good candidate to observe macroscopic quantum tunneling (MQT) and macroscopic quantum superpositions.

We consider a system of $N$ locally interacting bosons confined to the rotating external potential $V$ corresponding to a ring with a thin cut (a barrier). We assume that the transverse dimension of the ring is small compared to the healing length of the bosons and therefore the system can be considered effectively one-dimensional. The Hamiltonian of such system can be written as [3]

$$
\hat{H}=\sum_{n=1}^{N}\left[\frac{\left(i \nabla_{n}\right)^{2}}{2 m}+\Omega R \cdot i \nabla_{n}+V\left(r_{n}\right)\right]+\frac{\lambda}{2} \sum_{n \neq m}^{N} \delta\left(r_{n}-r_{m}\right)
$$

where $\Omega$ rotation frequency of the ring (i.e., the barrier), $R$ is the radius of the ring, $\lambda=4 \pi a / S m$, where $a$ is scattering length and $S$ is the ring's cross section area. Here and in the following we will use units with $\hbar=1$.

We start by evaluating the energy of the ground state of the Hamiltoian (1) using Gross-Pitaevskii approach. That is, we assume that the ground state wavefunction is a product, $\Psi\left(r_{1}, \ldots, r_{N}\right)=\chi\left(r_{1}\right) \ldots \chi\left(r_{N}\right)$ and minimize the the functional $E=\int d r_{1} \ldots d r_{N} \Psi^{*} H \Psi$ by varying it with respect to the single-particle state $\chi$. It is obvious, however, that, since the system is homogenous everywhere except in the small region at the barrier, 
$|\chi(r)|^{2} \simeq$ const or $\chi \sim e^{i \Phi(r)}$ (except in the vicinity of the barrier). Moreover, the phase-dependent terms in $E$ are $\sim \int d r\left[(\nabla \Phi)^{2} /(2 m)+\Omega R \nabla \Phi\right]$, and therefore $\Phi$ must be a linear function of distance along the ring, i. e., $\chi \sim e^{i \phi \theta / 2 \pi}$, where $\phi$ is the phase difference across the barrier and $\theta$ is the azimuthal angle parameterizing position along the ring. Evaluation of $E$ for such product state yields $N \phi^{2} / 2 m L^{2}-N \Omega \phi / 2 \pi+\lambda N(N-1) / 2 L$, where $L$ is the circumference of the ring. This expression, obviously, does not account for the contribution due to the barrier, i.e., the Josephson energy. At the barrier the particle density, $N|\chi(r)|^{2}$ is strongly dependent on $r$ and therefore must be calculated self-consistently. It can be shown, however, that to a good approximation, contribution of the barrier region into the system's energy can be cast in the form $-E_{\mathcal{J}} \cos \phi$, where the Josephson energy $E_{\mathcal{J}}$ is independent on $\phi[9]$. Thus we find that

$$
E(\phi)=\lambda N(N-1) / 2 L+N\left(\phi-\phi_{0}\right)^{2} / 2 m L^{2}-E_{\mathcal{J}} \cos \phi,
$$

where $\phi_{0}=m L^{2} \Omega / 2 \pi$.

When $\phi_{0}=\pi$ the effective potential is a symmetric double well, corresponding to two macroscopically different states, i. e., carrying different persistent currents. One can see, however, that such degeneracy is lifted by quantum fluctuations, leading to macroscopic quantum tunneling. In order to see this, let us evaluate the system's energy for the superposition state $C \int d \phi \Psi_{\phi}^{(0)}$, where $\Psi_{\phi}^{(0)}$ is the above product state and $C$ is normalization constant. A straightforward calculation shows that the expectation value of the interaction energy, i.e., of the last term in Eq. (11), is lower by $\lambda N / 2 L$ than that for the localized (in $\phi$ ) state $\Psi_{\phi}^{(0)}$.

We note, however, that while the interaction part of the Hamiltonian in Eq. (11) favors superposition, the first two terms , obviously, "prefer" the localized state. Therefore we search an optimal ground state wavefunction in the following form [10, 11]:

$$
\Psi\left(\mathbf{r}_{1}, \ldots, \mathbf{r}_{N}\right)=\sqrt{N / 24 \pi} \int d \phi \psi(\phi) \Psi_{\phi}^{(0)}\left(\mathbf{r}_{1}, \ldots, \mathbf{r}_{N}\right),
$$

where $\psi(\phi)$ is to be defined by minimization. In order to evaluate expectation value of the energy for the wavefunction in Eq. (3) we note that the states $\Psi_{\phi}^{(0)}$ are approximately orthogonal. Indeed, $\delta_{N}\left(\phi-\phi^{\prime}\right) \equiv$ $\int d \mathbf{r}_{1} \ldots d \mathbf{r}_{N} \Psi_{\phi}^{(0) *} \Psi_{\phi^{\prime}}^{(0)}$ yields $\delta_{N}(\phi)=[\sin (\phi / 2) / \phi / 2]^{N} \approx$ $e^{-N \phi^{2} / 24}$. This is a rapidly varying function - the combination $\sqrt{N / 24 \pi} \delta_{N}(\phi)$ approaches the true $\delta$-function for $N \gg 1$. Moreover, one can see that for any few-particle operator $\hat{A}$ the following identity holds:

$$
\left\langle\Psi_{\phi}^{(0)}|\hat{A}| \Psi_{\phi^{\prime}}^{(0)}\right\rangle=\delta_{N}\left(\phi-\phi^{\prime}\right)\left\langle\Psi_{\phi}^{(0)}|\hat{A}| \Psi_{\phi}^{(0)}\right\rangle[1+\varnothing(1 / N)] .
$$

As the result, one could think that the expectation value of (11) calculated with respect to the wavefunction (3) is merely $\int d \phi|\psi(\phi)|^{2}\left\langle\Psi_{\phi}^{(0)}|\hat{H}| \Psi_{\phi}^{(0)}\right\rangle=\int d \phi|\psi(\phi)|^{2} E(\phi)$. This, however, is not the case. The expectation value of the interaction part of the Hamiltonian is amplified by $N^{2}$ and the neglected $\varnothing(1 / N)$ terms become importantthe terms appearing due to the finite width of $\delta_{N}(\phi)$ contribute to the first order in $N$. A more careful evaluation of the interaction expectation value gives

$$
\frac{\lambda N(N-1)}{2 L} \int d \phi d \phi^{\prime} \psi\left(\phi^{\prime}\right)^{*} \psi(\phi) \sqrt{\frac{N}{24 \pi}} \delta_{N}\left(\phi^{\prime}-\phi\right) \frac{\left(\phi^{\prime}-\phi\right) / 2}{\tan \frac{\phi^{\prime}-\phi}{2}} .
$$

The finite width of $\delta_{N}\left(\phi^{\prime}-\phi\right)$ is clearly non-negligible. We use the identity $N^{2} \phi^{2} \delta_{N}(\phi) / 12=12 \partial_{\phi}^{2} \delta_{N}(\phi)+$ $N \delta_{N}(\phi)$ and evaluate the integral over $\phi$ containing $\partial_{\phi} \partial_{\phi} \delta_{N}\left(\phi^{\prime}-\phi\right)$ by parts, finally arriving at the result correct to $\varnothing(N)$

$$
E=-\frac{\lambda N}{2 L}+\int d \phi \psi^{*}(\phi)\left[-\frac{6 \lambda}{L} \partial_{\phi}^{2}+E(\phi)\right] \psi(\phi) .
$$

The first term in the right hand side of Eq. (6) is the negative offset mentioned earlier. The second term is a positive gain in energy due to variation of $\phi$. Together with the last term in Eq. (6) it can be viewed as kinetic and potential energies of a "phase-particle", whose dynamics obeys Schrodinger equation $\hat{H}_{\text {eff }} \psi=E \psi$, where $\hat{H}_{\mathrm{eff}}=-(6 \lambda / L) \partial_{\phi}^{2}+E(\phi)$.

The ground state for such "particle" is the symmetric superposition of two states in each well of the effective potential $E(\phi)$, separated from the next excited state, i.e., the antisymmetric combination, by the tunnel splitting

$$
\Delta E \sim \frac{N}{m L^{2} \eta} \exp \left[-\eta \sqrt{\alpha} \delta \phi^{3} / 72\right], \quad \delta \phi=\phi_{R}-\phi_{L},
$$

where the exponential prefactor is the frequency of small oscillation in each well, $\phi_{L}$ and $\phi_{R}$ are positions of each minima, $\eta=\sqrt{N S / 4 \pi a L}$ is Tonks parameter 12. (typically $\eta=10-100$ for cold atom systems), and $\alpha=m E_{\mathcal{J}} L^{2} / N$. When potential barrier is small, $\alpha \simeq 1$ 13. Energy $\Delta E$ defines the timescale of tunneling transitions between states with two different persistent currents.

The states with different persistent currents can be readily detected by looking at absorbtion images of the density distributions in time-of-flight (TOF) measurements [7, 8], e.g. Fig. (1). For long TOF $t_{0}$ single particle wavefunctions $\chi_{\phi}^{T O F}(\mathbf{r})$ become Fourier transforms of the initial single particle wavefunctions, $\chi_{\phi}^{T O F}(\mathbf{r}) \sim$ $\left.\chi_{\phi}(\mathbf{q})\right|_{\mathbf{q}=2 m \mathbf{r} / t_{0}}$. The outcome of the measurement is best understood by considering different moments of $\rho(\mathbf{q})$. A straightforward calculations yields

$$
\begin{aligned}
\langle\hat{\rho}(\mathbf{q})\rangle & =\int d \phi|\psi(\phi)|^{2} \rho_{\phi}(\mathbf{q}) \\
\left\langle\hat{\rho}(\mathbf{q}) \hat{\rho}\left(\mathbf{q}^{\prime}\right)\right\rangle & =\int d \phi|\psi(\phi)|^{2} \rho_{\phi}(\mathbf{q}) \rho_{\phi}\left(\mathbf{q}^{\prime}\right)
\end{aligned}
$$




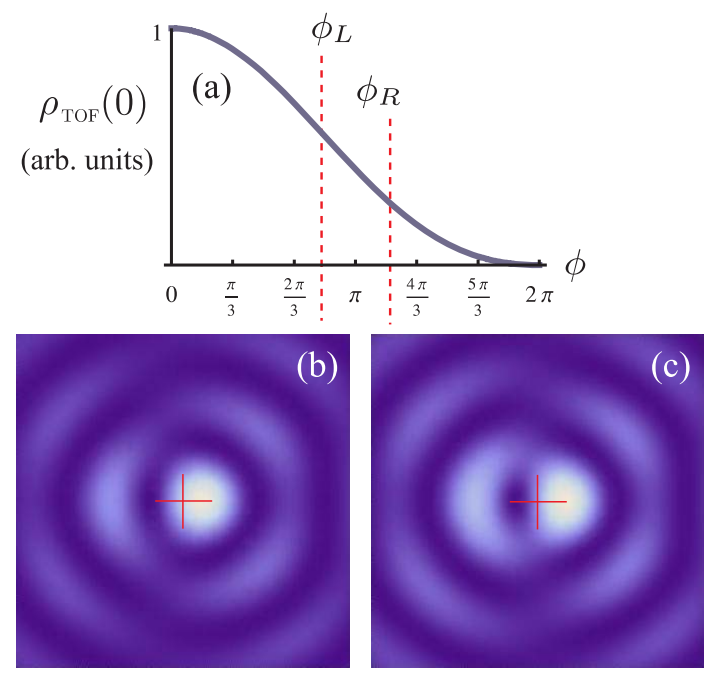

FIG. 1: (Color online) Expected TOF images of the Josephson-ring system in a double-well regime. (a) TOF density at the center as a function of $\phi$. Typical double-well potential minima $\phi_{L}, \phi_{R}$ are shown to give relevant scale on $\phi$. The typical TOF image corresponding to measurement outcome (b) $\phi_{L}$ and (c) $\phi_{R}$. In both cases the cross shows the center of the image.

where $\rho_{\phi}(\mathbf{q})=(N / S)\left|\chi_{\phi}(\mathbf{q})\right|^{2}$. These moments correspond to a stochastic process: the probability to observe the outcome $\rho_{\phi}\left(2 m \mathbf{r} / t_{\mathrm{TOF}}\right)$ and, hence, a particular value of $\phi$, is given by $|\psi(\phi)|^{2}$. Therefore, the measurement "chooses" a single term in the sum (3), and the function $\psi(\phi)$ becomes a quantum-mechanical wave function of a macroscopic object - the superfluid current of the entire condensate.

The above picture obviously is an approximation: We have assumed that all particles are "in the condensate", e.g., Eq. (3). For weakly interacting bosons the ground state is no longer a product, but the well-known Bogolubov state. Therefore we consider a modified ground state wavefunction, still in the form of Eq. (3), but with modified $\Psi_{\phi}^{(0)}$, i.e.,

$$
\left|\Psi_{\phi}^{(0)}\right\rangle \rightarrow\left|\Psi_{\phi}\right\rangle \sim\left(\sum_{n} c_{n} a_{\phi+2 \pi n}^{\dagger} a_{\phi-2 \pi n}^{\dagger}\right)^{N / 2}|0\rangle,
$$

where $a_{\phi+2 \pi n}^{(\dagger)}$ is a creation (annihilation) operator corresponding to a single-particle state $\chi_{\phi, n}(\mathbf{r})=\chi_{\phi}(\mathbf{r}) e^{i n \theta}$, and $c_{n}$ are variational coefficients [14]. By choosing the trial wavefunction in the form of Eq. (9) we assume that the dynamics is adiabatic: The slow collective variable $\phi$ is coupled to fast degrees of freedom, i.e., the fluctuations. Indeed, due to the finite size of the the system the quasiparticle spectrum is discrete with gaps $\sim($ speed of sound $/ L)=N /\left(m L^{2} \eta\right)$, which is much greater than the energy scale associated with the tunneling between two wells, e.g., Eq. (7). As a result the quasiparticles remain in the ground state and readjust to the variations in $\phi$.

Corrections to the effective energy functional, e.g., Eq. (6), turn out to be small in the limit of weak interactions. The new ground state energy can be evaluated by using Eq. (4), which, as can be directly verified, still holds for the modified wavefunction, e.g., Eq. (9). Then, evaluating $\left\langle\Psi_{\phi}|\hat{H}| \Psi_{\phi}\right\rangle$ and varying $c_{n}$ 's (see Ref. [3] for similar calculation) we find that corrections to the functional $E$ in Eq. (6) are suppressed by factor $1 / \eta$, i.e., are small in the weak interaction limit [15].

The effect of fluctuations, however, turns out to be quite appreciable as far as measurement of close currentcarrying states is concerned. Indeed, while the number of non-condensate particles is small, the difference between density profiles (in the TOF measurements) corresponding to different values of $\phi$, i.e., different persistent currents, is also small. In the opposite case the barrier is to high to allow for tunneling on a reasonable time scale. Moreover, as we will see, both the tunneling exponent in Eq. (7) and the signal-to-noise ratio due to "non-condensate" particles (to be defined below) are controlled by the same parameters. To see this let us evaluate the set of correlation functions as in Eq. (8), but for the modified wavefunction given by Eq. (9). In doing so we again use Eq. (4), and so the calculation reduces to the evaluation of averages $\left\langle\Psi_{\phi}\left|\hat{\rho}(q) \hat{\rho}\left(q^{\prime}\right) \ldots\right| \Psi_{\phi}\right\rangle$, where TOF density operator $\hat{\rho}(q)=\sum_{n, n^{\prime}} \chi_{\phi, n}^{*}(q) \chi_{\phi, n^{\prime}}(q) a_{n}^{\dagger} a_{n^{\prime}}$ (again $\chi_{\phi, n}(q)$ is the Fourier transform of $\chi_{\phi, n}(r)$ ). The first two moments are

$$
\begin{aligned}
\langle\hat{\rho}(\mathbf{q})\rangle & =\int d \phi|\psi(\phi)|^{2} \tilde{\rho}_{\phi}(\mathbf{q}) \\
\left\langle\hat{\rho}(\mathbf{q}) \hat{\rho}\left(\mathbf{q}^{\prime}\right)\right\rangle & =\int d \phi|\psi(\phi)|^{2}\left[\tilde{\rho}_{\phi}(\mathbf{q}) \tilde{\rho}_{\phi}\left(\mathbf{q}^{\prime}\right)+\Lambda_{\phi}\left(\mathbf{q}, \mathbf{q}^{\prime}\right)\right]
\end{aligned}
$$

where $\tilde{\rho}_{\phi}(\mathbf{q})=\rho_{\phi}(\mathbf{q})+\sum_{n \neq 0}\left\langle a_{\phi, n}^{\dagger} a_{\phi, n}\right\rangle\left|\chi_{\phi, n}(\mathbf{q})\right|^{2}$ is the renormalized density and

$$
\begin{aligned}
\Lambda_{\phi}\left(\mathbf{q}, \mathbf{q}^{\prime}\right) & =N \sum_{n}\left[\chi_{\phi, n}^{*}(\mathbf{q}) \chi_{\phi, n}\left(\mathbf{q}^{\prime}\right)\left\langle a_{\phi+2 \pi n}^{\dagger} a_{\phi+2 \pi n}\right\rangle\right. \\
& \left.+\chi_{\phi, n}(\mathbf{q}) \chi_{\phi, n}\left(\mathbf{q}^{\prime}\right)\left\langle a_{\phi+2 \pi n} a_{\phi+2 \pi n}\right\rangle+\text { c. c. }\right] .
\end{aligned}
$$

In evaluating Eqs. (10, 11) we have used Wick's theorem as well as the fact that in the limit of weak interactions $\left.\left.\left\langle a_{\phi+2 \pi n}^{\dagger} a_{\phi+2 \pi n}\right\rangle\right|_{n=0} \simeq N \gg\left\langle a_{\phi+2 \pi n}^{\dagger} a_{\phi+2 \pi n}\right\rangle\right|_{n \neq 0}$ and therefore products $\left\langle a_{\phi+2 \pi n}^{\dagger} a_{\phi+2 \pi n}\right\rangle\left\langle a_{\phi+2 \pi n^{\prime}}^{\dagger} a_{\phi+2 \pi n^{\prime}}\right\rangle$, etc., with $n, n^{\prime} \neq 0$, can be neglected. Moreover, since $\Lambda_{\phi}\left(\mathbf{q}, \mathbf{q}^{\prime}\right)$ is small compared to $\tilde{\rho}_{\phi}(\mathbf{q}) \tilde{\rho}_{\phi}\left(\mathbf{q}^{\prime}\right)$, we can replace it by $\Lambda_{\pi}\left(\mathbf{q}, \mathbf{q}^{\prime}\right)$ in Eq. (10), - we are interested in the situation when the barrier separating the two persistent currents is small, $\delta \phi \ll \pi$. As a result $\Lambda_{\pi}\left(\mathbf{q}, \mathbf{q}^{\prime}\right)$ can be viewed as the correlation function of noise superimposed with "signal" $\tilde{\rho}_{\phi}(\mathbf{q})$. It is natural, therefore, to introduce a signal to noise ratio (SNR) as

$$
\mathrm{SNR}=\frac{\left[\rho_{\pi-\delta \phi / 2(0)}-\rho_{\pi+\delta \phi / 2}(0)\right]^{2}}{\Lambda_{\pi}(0,0)}
$$


where we define the strength of the signal as the difference in the TOF particle densities at the center for two realizations with phases $\pi \pm \delta \phi$, e.g., Fig. 1 . The SNR in Eq. (12) can be easily evaluated: At the center $\chi_{\phi, n}(\mathbf{q}=$ $0)=B /(\phi / 2 \pi+n)$, where $B=(1 / 2 \pi) \int d \mathbf{r} \chi_{\pi}(\mathbf{r})$. Then we obtain that

$$
\Lambda_{\pi}(0,0)=\frac{4 N^{2} B^{4}}{\pi^{4}} \sum_{n \neq 0}\left[\frac{\left\langle a_{n}^{\dagger} a_{n}\right\rangle}{1-4 n^{2}}+\frac{\left\langle a_{n} a_{-n}\right\rangle}{(1+2 n)^{2}}+c . c .\right]
$$

where a shortcut $a_{n}^{(\dagger)}=a_{\pi+2 \pi n}^{(\dagger)}$ has been used to reduce notations. The averages in Eqs. (13) can be found from the normal and anomalous Green's functions of the interacting boson system, i.e. $\left\langle a_{n}^{\dagger} a_{n}\right\rangle=$ $\int \frac{d \omega}{2 \pi} \mathcal{G}(n, i \omega)$ and $\left\langle a_{n} a_{-n}\right\rangle=\int \frac{d \omega}{2 \pi} \mathcal{F}(n, i \omega)$, where $\mathcal{G}(n, i \omega)=\frac{-i \omega-\epsilon_{n}^{\phi}}{\omega^{2}+\left(\varepsilon_{n}^{\phi}\right)^{2}}$ and $\mathcal{F}(n, i \omega)=\frac{\lambda \rho / 2}{\omega^{2}+\left(\varepsilon_{n}^{\phi}\right)^{2}}$, with $\epsilon_{n}^{\phi}=\hbar^{2}\left(\phi-\phi_{0}+2 \pi n\right) / 2 m L^{2}+\lambda \rho / 2$ and $\varepsilon_{n}^{\phi}=$ $\sqrt{\left(\epsilon_{n}^{\phi}\right)^{2}-(\lambda \rho / 2)^{2}}[16]$. After straightforward calculation we find that

$$
\mathrm{SNR} \simeq 7.84 \eta \delta \phi^{2}
$$

Eq. (14) is the principal result of this paper. It reflects the fundamental difference between the phase dynamics of the Josephson qubits and single body quantum mechanics: While quantum mechanics, in principle, allows one to measure particle's coordinate with infinite precision, the accuracy of the phase measurements in Josephson qubits is limited by their many-body nature. Remarkably, the precision for such measurement is controlled by a single parameter $\eta$, i.e., the interaction strength. Moreover, comparing Eq. (17) with Eq. (14) we see that both the tunneling exponent and the SNR are controlled by the same parameters. This fact is not surprising: while stronger interactions enhance MQT, e.g., Eq. (7), they decrease the SNR due to the suppression of the condensate density for stronger scattering. The tunneling exponent, however, increases faster (in absolute value) with the growth of $\delta \phi$ and therefore to keep it small together with the condition SNR $\gg 1$ we need $\eta \gg 1$. Thus coherent MQT is observable only in weakly interacting BEC systems. For typical BEC experiment $\eta \sim 10-100$ [7, 8, 17]. If we keep the tunneling exponent in (17) equals to $\sim 1$ we obtain $\mathrm{SNR} \sim 300-600$.

To conclude, we have demonstrated that a cold atom Josephson-ring system can be described by an effective single-particle Schrodinger equation with an effective potential that can be controlled by the system's rotation. We analyzed the statistics of the TOF measurements that allow one to determine the system's current, i.e., phase across the Josephson junction. We found that fidelity of such measurements is limited by the ground state fluctuations and is controlled by the Tonks parameter.

Finally we conjecture that similar limitations are likely to take place in superconducting flux qubits. While nu- merous studies of such systems have been carried out, including the derivation of the effective Schrodinger equation, etc., generalization of the results obtained in this paper to the superconducting case, however, does not seem to be straightforward due to a different nature of the ground state for a fermionic system. Thus we believe that the extension of our results to superconductors is an interesting direction for future work.

We thank M. G. Boshier, A. J. Leggett, I. Martin, V. Privman and E. Timmermans for valuable discussions and comments. The work is supported by the US DOE.

[1] Andrews, M. R., H-J. Miesner, D. M. Stamper-Kurn, J. Stenger, and W. Ketterle, Phys. Rev. Lett. 82, 2422 (1999).

[2] S. Levy, E. Lahoud, I. Shomroni, and J. Steinhauer, Nature 449, 579 (2007).

[3] A. J. Leggett, Rev. Mod. Phys. 73, 307 (2001).

[4] D. R. Dounas-Frazer, A. M. Hermundstad, and L. D. Carr, Phys. Rev. Lett. 99, 200402 (2007).

[5] B. P. Anderson, K. Dholakia, and E. M. Wright, Phys. Rev. A 67, 033601 (2003); S. Gupta, K. W. Murch, K. L. Moore, T. P. Purdy, and D. M. Stamper-Kurn, Phys. Rev. Lett. 95, 143201 (2005); C. Ryu, M. F. Andersen, P. Clade, Vasant Natarajan, K. Helmerson, and W. D. Phillips, Phys. Rev. Lett. 99, 260401 (2007).

[6] H. P. Buchler, V. B. Geshkenbein, and G. Blatter, Phys. Rev. Lett. 87, 100403 (2001); N. Didier, A. Minguzzi, and F. W. J. Hekking, Phys. Rev. A 79, 063633 (2009).

[7] D. Solenov, D. Mozyrsky, Phys Rev. Lett. 104, 150405 (2010).

[8] K. Henderson, C. Ryu, C. MacCormick and M. G. Boshier, New J. Phys. 11, 043030 (2009).

[9] F. Dalfovo, L. Pitaevskii, amd S. Stringari, Phys. Rev. A 54, 4213 (1996); I. Zapata, F. Sols and A. J. Leggett, Phys. Rev. A 57, R28 (1998).

[10] This ansatz was first proposed by Leggett in the context of superconducting Josephson rings [1]

[11] A. J. Leggett, Quantum mechanics at the macroscopic level, Ecole d'été de physique théorique (Les Houches, Haute-Savoie, France) (46th: 1986)

[12] D. S. Petrov, G. V. Shlyapnikov, and J. T. M. Walraven, Phys. Rev. Lett. 85, 3745 (2000).

[13] The barrier separating two wells disappears when $\alpha=1$.

[14] For a detailed discussion of Bogolubov's ground state wavefunction see Ref. [3] and references therein.

[15] The account of fluctuations, e.g., Eq. (9), obviosuly leads to lower ground state energy. The $\phi$-independent correction is of the order of $-\lambda N^{2} /(L \eta)$, while the leading $\phi$ dependent contribution is $-N\left(\phi-\phi_{0}\right)^{2} /\left(4 \pi \sqrt{2} \eta m L^{2}\right)$.

[16] A. A. Abrikosov, L. P. Gorkov, and I. E. Dzyaloshinski, Methods of quantum field theory in statistical physics (Dover, 1975).

[17] E. A. Donley, N. R. Claussen, S. L. Cornish, J. L. Roberts, E. A. Cornell, C. E. Wieman, Nature 412, 295 (2001). 